\title{
Influência de Alguns Fatores de Ambiente sobre os Escores de Conformação, Precocidade e Musculatura à Desmama em um Rebanho da Raça Nelore ${ }^{1}$
}

\author{
João Jorge Júnior², Fabiano Veraldo da Costa Pita ${ }^{3}$, Luiz Alberto Fries ${ }^{4}$, Lucia Galvão de Albuquerque ${ }^{5}$
}

RESUMO - Dados de 23.251 animais da raça Nelore desmamados no período de 1994 a 1997, pertencentes à Agropecuária Jacarezinho Ltda., foram analisados para se avaliar os efeitos ambientais da idade da vaca, data de nascimento e idade à desmama sobre os escores visuais de conformação (C), precocidade (P) e musculatura (M). Todos os efeitos estudados influenciaram significativamente os escores visuais. As análises indicaram que as vacas que pariram aos sete, oito e nove anos, obtiveram os melhores resultados para os escores visuais de C, P e M, assim como os animais que nasceram mais cedo dentro da estação de parição. Para a idade à desmama foi constatado que, quanto mais tarde o animal for desmamado, melhores serão seus escores. Os animais que desmamaram com 220 dias tiveram os melhores escores, enquanto os piores escores foram daqueles que desmamaram com 140 dias. Portanto, para se ter maior precisão na avaliação genética dos indivíduos jovens, fatores de correção para idade da vaca, data juliana de nascimento e idade à desmama devem ser utilizados sobre os escores de conformação, precocidade e musculatura.

Palavras-chave: data juliana de nascimento, escores visuais, gado de corte, idade do bezerro à desmama, idade da vaca

\section{Influence of Some Environmental Effects on Visual Scores of Conformation, Precocity and Musculature Measured at Weaning in Nelore Cattle}

\begin{abstract}
Data on 23,251 Nelore calves weaned from 1994 to 1997, belonging to Agropecuária Jacarezinho Ltda., were analyzed to estimate the influence of environmental effects of age of dam, julian date of birth and age of calf at weaning on visual scores of conformation (C), precocity (P) and musculature (M). All effects significantly influenced the visual scores. The analyses showed that the calves with the best scores of C, P and $\mathrm{M}$ at weaning were those from dams with 7, 8 and 9 years of age. The best visual scores were from animals that were born earlier in the calving season. For age at weaning, as older was the animal better were its scores. The highest scores were those from animals weaned at 220 days of age and the smallest scores were from animals weaned at 140 days of age. Therefore, to increase the accuracy of genetic evaluations of young animals, adjusting factors for age of dam, julian date of birth and age at weaning, should be used, for conformation, precocity and musculature scores.
\end{abstract}

Key Words: beef cattle, calf age, cow age, julian date of birth, visual score

\section{Introdução}

Em gado de corte, a seleção dos animais para peso ou ganhos em peso é de fundamental importância e sua utilização deverá trazer benefícios econômicos significativos para os produtores e para o país. Segundo FRISCH (comunicação pessoal), o ganho de peso pós desmama, a pasto, durante 12 meses, é o melhor indicador da capacidade de adaptação dos animais.

Entretanto, no Brasil, alguns núcleos têm realizado seleção por vários anos, quase que exclusivamente para peso, inclusive com grande valorização de animais com alto peso adulto. Como os pesos nas diferentes idades são geneticamente correlacionados, a seleção a longo prazo exclusivamente para peso em diferentes idades tem levado a um aumento do peso adulto ocasionando, em certas regiões do país, um desequilíbrio entre as exigências de mantença e a disponibilidade de matéria seca. Como conseqüência, tem-se produzido animais grandes e tardios, que não estão de acordo com o sistema de criação a pasto, mais freqüentemente empregado no país.

A tendência atual, na busca de animais precoces, que atendam as exigências do mercado em termos de quantidade e qualidade de carne e de equilíbrio com o sistema de produção, tem mostrado a necessidade de mudanças nos critérios de seleção. Diante disso, o

\footnotetext{
${ }^{1} \mathrm{CNPq}$.

${ }^{2}$ Aluno do curso de pós-graduação em Genética e Melhoramento Animal. (Bolsista de mestrado-FAPESP) - DZ-FCAV-UNESP E.mail: jjorge@fcav.unesp.br

${ }^{3}$ Aluno do curso de pós-graduação em Genética e Melhoramento (Doutorado) - UFV. E.mail: fvcpita@bol.com.br

${ }^{4}$ Gensys Consultores Associados S/C Ltda.

${ }^{5}$ Professora Assistente Doutora - DZ-FCAV-UNESP. Pesquisadora do CNPq. E.mail: Igalb@fcav.unesp.br
} 
que tem sido proposto é selecionar animais não pensando no peso como característica única a ser explorada, mas também nas precocidades sexual, de crescimento e de terminação, aliadas aos índices reprodutivos e à qualidade da carcaça.

Dentro deste pensamento é que se estabeleceu o uso de escores visuais, primeiramente nos EUA, através do sistema de avaliação Ankony (LONG, 1973), que se baseia numa escala absoluta de 1 a 10 para algumas características medidas visualmente. No Brasil Central, diversos grupos estão usando a avaliação visual para características como conformação $(\mathrm{C})$, precocidade $(\mathrm{P})$ e musculatura $(\mathrm{M})$ para compor um índice geral do animal, utilizado posteriormente como critério de seleção.

Apesar de estarem sendo estimadas DEP's para escores visuais e a qualidade desta estimativa depender dos efeitos ambientais que influenciam estas características, poucos estudos tem sido realizados no sentido de avaliar os fatores de meio que afetam $\mathrm{C}, \mathrm{P}$ e $\mathrm{M}$ em bovinos de corte.

Assim, o objetivo deste trabalho foi avaliar a influência de alguns fatores de ambiente sobre os escores corporais de conformação, precocidade e musculatura, avaliados à desmama, em um rebanho da raça Nelore.

\section{Material e Métodos}

Foram utilizados escores visuais de conformação, precocidade e musculatura, registrados à desmama, de 23.251 animais Nelore, pertencentes à Agropecuária Jacarezinho Ltda., nascidos entre 1993 e 1996. A conformação (C) é avaliada através da classificação do animal, em termos de capacidade de produção de carne, se abatido naquele momento, considerando-se a combinação da quantidade de carne na carcaça e a presença de massas musculares. Para precocidade (P) são observadas as formas das massas musculares, pontuando-se os animais conforme a expectativa de se chegar a um acabamento mínimo de carcaça com peso vivo não elevado. Os animais com boa precocidade de terminação são aqueles que apresentam boa abertura torácica, boa profundidade de costelas, boas massas musculares e virilha pesada, aliada a um bom desenvolvimento corporal. No que se refere à musculatura $(\mathrm{M})$, a presença de massas musculares principalmente nos quartos traseiros, no lombo, paleta e antebra- ço são levadas em consideração. As notas para essas três características variam de 1 a 5 , sendo 5 a nota máxima. Os escores não são atribuídos numa escala absoluta, como é o usual por exemplo, no sistema Ankony de LONG (1973). Neste conjunto de dados, primeiro o avaliador observa o grupo em conjunto e estabelece o animal 3 (média do grupo contemporâneo) e os extremos 1 e 5, para cada um dos escores visuais, dentro daquele grupo. A seguir, cada animal é avaliado individualmente e os escores são atribuídos em relação ao seu grupo contemporâneo.

A estação reprodutiva teve duração de 70 dias para as vacas e 60 dias para as novilhas. Isto é feito para aumentar a pressão de seleção no início da vida reprodutiva e evitar partos tardios. A estação das novilhas de 18 meses (precoces) é realizada entre os meses de abril e junho.

As novilhas que emprenharam aos 18 meses, pariram nos meses de janeiro, fevereiro e março e fazem parte das chamadas precoces, as quais foram tratadas à parte no programa de melhoramento. Todos os seus demais serviços se deram na primavera. Por outro lado, as progênies das novilhas servidas no outono tiveram todos seus serviços na primavera, o primeiro serviço também aos 18 meses. Dessa forma, espera-se que na primeira estação de parição haja um confundimento entre os efeitos de idade da vaca e de época de nascimento.

Os fatores ambientais avaliados foram: idade da vaca ao parto, época de nascimento e idade do bezerro à desmama, considerados em classes. A idade da vaca variou de 2 a 19 anos, sendo que aquelas que apresentaram pequeno número de observações foram eliminadas, restando então vacas entre 3 e 14 anos. A data de nascimento variou de $1^{\circ}$ de janeiro a 21 de março para as novilhas e de 10 de agosto a 7 de dezembro para as vacas. A idade de desmama variou de 141 a 259 dias. Foram formadas classes a cada 20 dias para idade do animal à desmama e data juliana de nascimento. Animais que possuíam classe de data juliana de nascimento entre 5 e 11 (22 de março a 9 de agosto) e maior que 17 ( 8 a 31 de dezembro) foram eliminados, o mesmo ocorrendo com animais de classe de idade à desmama igual a 6 (entre 121 e 140 dias) e maior que 12 (maior que 259 dias), devido ao pequeno número de observações. 
O número de observações e as médias observadas para os escores visuais de acordo com a Idade da Vaca ao Parto, Classes de Data Juliana de Nascimento, Idade do Bezerro à Desmama, estão apresentadas nas Tabelas 1,2 e 3, respectivamente.

Os escores visuais foram analisados separadamente, através do método dos Quadrados Mínimos, empregando-se o procedimento GLM (SAS, 1995).

O modelo estatístico empregado pode ser representado, na forma matricial, como:

$$
\mathbf{y}=\mathbf{X} \boldsymbol{\beta}+\mathbf{e}
$$

em que: $\mathbf{y}=$ vetor de $\operatorname{dados}(\mathrm{C}, \mathrm{P}$ ou $\mathrm{M})$; $\mathbf{X}=$ matriz de incidência; $\boldsymbol{\beta}=$ vetor de efeitos fixos; $\mathbf{e}=$ vetor de resíduos.

$$
\mathrm{E}(\mathbf{y})=\mathbf{X} \boldsymbol{\beta} ; \mathrm{E}(\mathbf{e})=\mathbf{0} \text { e } \operatorname{Var}(\mathbf{e})=\mathbf{I} \boldsymbol{\sigma}_{\mathbf{e}}^{\mathbf{2}}
$$

em que: $\mathbf{I}=$ matriz identidade $\boldsymbol{\sigma}_{\mathbf{e}}^{\mathbf{2}}=$ componente de variância do resíduo.

Os efeitos fixos considerados foram grupo contemporâneo (GC), idade da vaca em anos, classe de data de nascimento e classe de idade à desmama. $\mathrm{O}$ GC foi constituído por fazenda, retiro, sexo, ano e mês de nascimento, grupo de manejo à desmama e data de desmama, tendo sido criados 356 grupos.

Tabela 1 - Número de observações $(\mathrm{N})$ e médias observadas para escores de conformação (C), precocidade $(\mathrm{P})$ e musculatura $(\mathrm{M})$ de bezerros, em função da idade da vaca ao parto (IDVACP)

Table 1 - Number of records $(N)$ and means for conformation, precocity and musculature visual scores, according to age of dam at parturition (ADP)

\begin{tabular}{ccccc}
\hline IDVACP & $\mathrm{N}$ & & \multicolumn{2}{c}{$\begin{array}{c}\text { Médias } \\
\text { Means }\end{array}$} \\
\cline { 3 - 5 } & $N$ & $\mathrm{C}$ & $\mathrm{P}$ & $\mathrm{M}$ \\
\hline 3 & & 3,17 & 3,24 & 3,13 \\
4 & 7239 & 3,12 & 3,25 & 3,10 \\
5 & 4748 & 3,22 & 3,35 & 3,22 \\
6 & 2382 & 3,48 & 3,57 & 3,43 \\
7 & 1845 & 3,57 & 3,65 & 3,54 \\
8 & 1482 & 3,52 & 3,60 & 3,50 \\
9 & 1459 & 3,55 & 3,65 & 3,55 \\
10 & 1170 & 3,51 & 3,58 & 3,49 \\
11 & 1010 & 3,53 & 3,65 & 3,54 \\
12 & 714 & 3,46 & 3,60 & 3,47 \\
13 & 519 & 3,37 & 3,45 & 3,31 \\
14 & 296 & 3,29 & 3,46 & 3,31 \\
\hline
\end{tabular}

Tabela 2 - Número de observações $(N)$ e médias observadas para escores de conformação $(C)$, precocidade $(P)$ e musculatura (M) de bezerros, em função das classes de data juliana de nascimento (CLDJN)

Table 2 - Number of records $(N)$ and means for conformation, precocity and musculature visual scores, according to Julian birth date

\begin{tabular}{|c|c|c|c|c|c|}
\hline \multirow[t]{2}{*}{$\begin{array}{l}\text { CLDJN } \\
J B D C\end{array}$} & \multirow[t]{2}{*}{$\begin{array}{l}\text { Datas julianas } \\
\text { Julian dates }\end{array}$} & \multirow[t]{2}{*}{$\begin{array}{l}\mathrm{N} \\
N\end{array}$} & \multicolumn{3}{|c|}{$\begin{array}{l}\text { Médias } \\
\text { Means }\end{array}$} \\
\hline & & & $\mathrm{C}$ & $\mathrm{P}$ & $\mathrm{M}$ \\
\hline 1 & $1^{\circ}$ janeiro -20 janeiro & 307 & 3,61 & 3,54 & 3,50 \\
\hline 2 & 21 janeiro - 9 fevereiro & 498 & 3,43 & 3,45 & 3,34 \\
\hline 3 & 10 fevereiro $-1^{\circ}$ março & 405 & 3,13 & 3,20 & 3,07 \\
\hline 4 & 2 março - 21 março & 74 & 3,43 & 3,58 & 3,34 \\
\hline 12 & 10 agosto - 29 agosto & 1097 & 3,92 & 3,98 & 4,00 \\
\hline 13 & 30 agosto -18 setembro & 6126 & 3,47 & 3,52 & 3,47 \\
\hline 14 & 19 setembro - 8 outubro & 6651 & 3,25 & 3,35 & 3,22 \\
\hline 15 & 9 outubro - 28 outubro & 4323 & 3,18 & 3,32 & 3,15 \\
\hline 16 & 29 outubro - 17 novembro & 2608 & 3,02 & 3,18 & 3,02 \\
\hline 17 & 18 novembro - 7 dezembro & 1162 & 2,95 & 3,12 & 2,92 \\
\hline
\end{tabular}
classes (JBDC) 
Tabela 3 - Número de observações $(\mathrm{N})$ e médias observadas para escores de conformação $(\mathrm{C})$, precocidade (P) e musculatura (M) de bezerros, em função das classes de idade do bezerro à desmama (CLIDD)

Table 3 - Number of records $(N)$ and means for conformation, precocity and musculature visual scores, according to age of calf at weaning classes (ACWC)

\begin{tabular}{lccccc}
\hline $\begin{array}{l}\text { CLIDD } \\
\text { ACWC }\end{array}$ & $\begin{array}{c}\text { Dias de idade } \\
\text { Days of age }\end{array}$ & $\mathrm{N}$ & \multicolumn{3}{c}{$\begin{array}{c}\text { Médias } \\
\text { Means }\end{array}$} \\
\cline { 4 - 6 } & & $N$ & $\mathrm{C}$ & $\mathrm{P}$ & $\mathrm{M}$ \\
\hline 7 & $141-159$ & 208 & 2,28 & 2,43 & 2,36 \\
8 & $160-179$ & 1878 & 2,83 & 2,94 & 2,84 \\
9 & $180-199$ & 12060 & 3,19 & 3,30 & 3,17 \\
10 & $200-219$ & 8233 & 3,53 & 3,62 & 3,51 \\
11 & $220-239$ & 747 & 3,63 & 3,69 & 3,56 \\
12 & $240-259$ & 125 & 3,65 & 3,57 & 3,44 \\
\hline
\end{tabular}

\section{Resultados e Discussão}

As médias gerais observadas para os escores visuais foram de 3,29; 3,39 e 3,27, respectivamente para C, P e M. A Tabela 4 apresenta um resumo das análises de variâncias para as três características estudadas. Todos os efeitos considerados no modelo afetaram significativamente $(\mathrm{P}<0,001)$ os escores de $\mathrm{C}, \mathrm{P}$ e $\mathrm{M}$.

Estes resultados estão de acordo com os de PONS (1989) e PONS et al. (1989), que encontraram efeito significativo da idade da vaca e data de nascimento sobre os escores de conformação. Da mesma forma, CARDOSO et al. (1997) encontraram efeito significativo da idade da vaca ao parto sobre C, P e $\mathrm{M}$, na raça Santa Gertrudis. Entretanto, estes autores observaram efeito significativo da idade do bezerro à desmama apenas para $\mathrm{C}$ e $\mathrm{M}$.
Com o objetivo de facilitar o entendimento e a visualização de cada efeito, para confecção das figuras, optou-se por somar a média geral de cada característica às soluções de quadrados mínimos correspondentes. As soluções de quadrados mínimos, de acordo com as classes de idade da vaca ao parto, podem ser observadas nas Figuras 1, 2 e 3, respectivamente para $\mathrm{C}, \mathrm{P}$ e $\mathrm{M}$. A idade da vaca ao parto, cuja média geral foi de 5,5 anos, apresentou efeito semelhante para as três características. Os escores visuais aumentaram com a idade da vaca até a mesma atingir entre 7 e 8 anos de idade, decrescendo a seguir. Vacas que pariram aos 7, 8 e 9 anos foram as que desmamaram os bezerros com os maiores escores de $\mathrm{C}, \mathrm{P}$ e $\mathrm{M}$, sendo que os melhores resultados foram obtidos com vacas que pariram aos 8 anos de idade. Por outro lado, vacas jovens (com menos de

Tabela 4 - Fontes de variação, graus de liberdade (GL) e quadrados médios para os escores de conformação (C), precocidade (P) e musculatura (M)

Table 4 - Sources of variation, degrees of freedom (DF) and means square for confomation (C), precocity (P) and musculature (M) scores

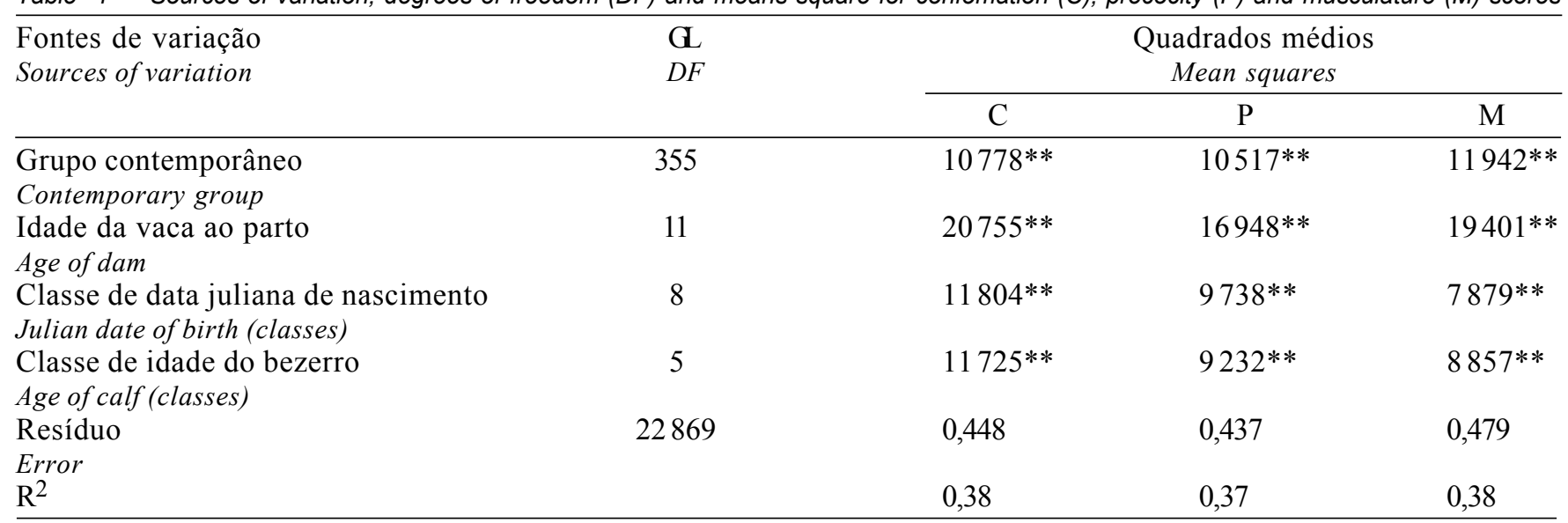

** Significativo a $0,1 \%$ de probabilidade, pelo teste $\mathrm{F}$.

** Significant at $0.1 \%$ of probability, by $F$ test.

Rev. bras. zootec., 30(6):1697-1703, 2001 


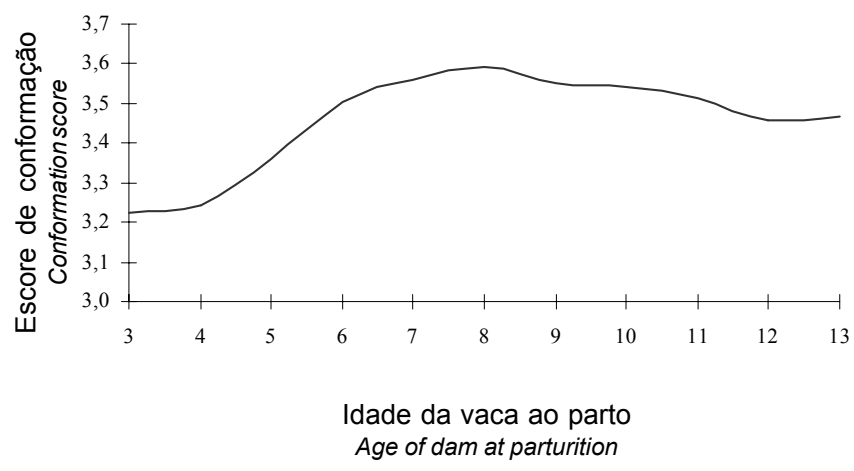

Figura 1 - Soluções de quadrados mínimos dos escores de conformação de bezerros $(+3,29)$, de acordo com a idade da vaca ao parto.

Figure 1 - Least square solutions for conformation scores of calves (+3.29), according to age of dam at parturition.

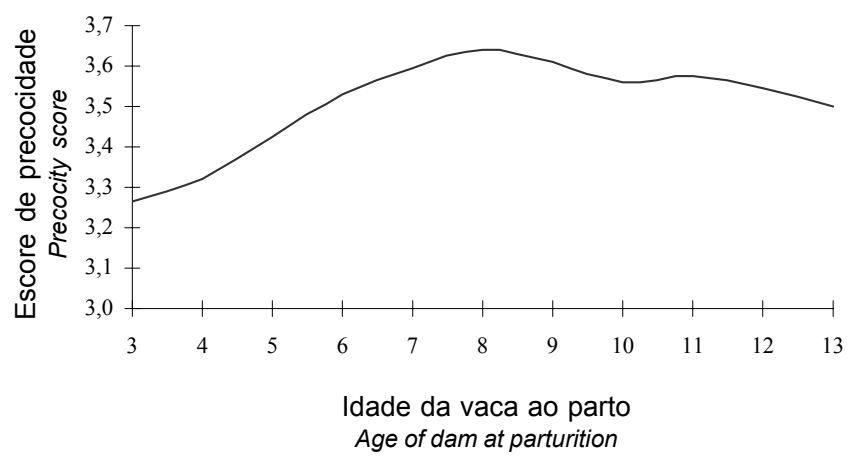

Figura 2 - Soluções de quadrados mínimos dos escores de precocidade de bezerros $(+3,39)$, de acordo com a idade da vaca ao parto.

Figure 2 - Least square solutions for precocity scores of calves (+3.39), according to age of dam at parturition.

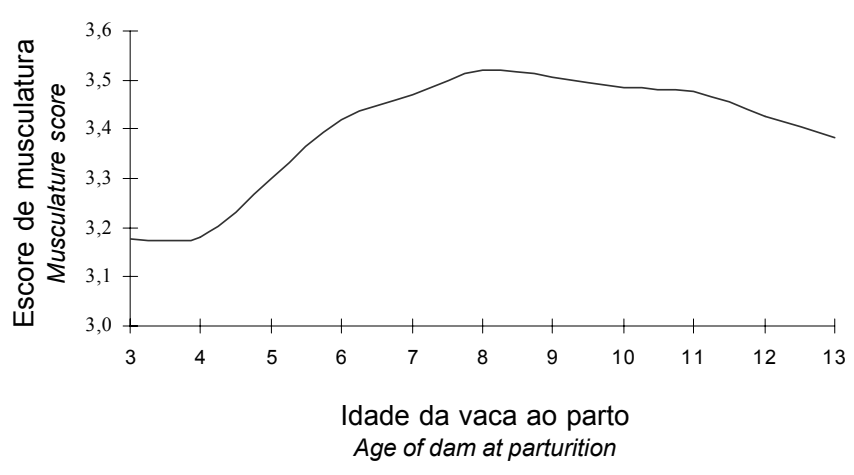

Figura 3 - Soluções de quadrados mínimos dos escores de musculatura de bezerros $(+3,27)$, de acordo com a idade da vaca ao parto.

Figura 3 - Least square solutions for musculature scores of calves (+3.27), according to age of dam at parturition.
6 anos), desmamaram bezerros com escores menores do que vacas que pariram com mais de 10 anos de idade.

Estes resultados são semelhantes aos de PONS (1989) para a raça Hereford, cujos escores de conformação foram crescentes com a idade da vaca, até 7 a 8 anos e decrescentes após esta idade. Da mesma forma, MOREIRA e CARDELLINO (1994) verificaram um aumento no escore de conformação com o aumento da idade da vaca, ocorrendo os maiores valores com vacas que pariram aos 8-9 anos, resultados também semelhantes aos observados por FRIES (1996a) e PAZ (1997), ao estudarem o efeito da idade da vaca sobre o peso e ganho de peso até a desmama.

A média observada para a data juliana de nascimento foi o $260^{\circ}$ dia, com animais nascidos do $1^{\circ}$ até o $80^{\circ}$ e de $222^{\circ}$ até $341^{\circ}$ dia do ano. As quatro primeiras classes de data juliana de nascimento (do dia $1^{\circ}$ de janeiro ao $21^{\circ}$ dia de março) apresentaram um pequeno número de observações. Estes animais são filhos de vacas que conceberam em torno dos 18 meses, fora da estação de monta normal.

As soluções de quadrados mínimos para C, P e M, de acordo com as classes de data juliana de nascimento, podem ser observadas nas Figuras 4, 5 e 6, respectivamente. De maneira geral, para as duas estações de nascimento, os animais nascidos mais cedo dentro da estação apresentaram escores visuais mais elevados para as três características. Provavelmente isto se deva ao fato dos mesmos serem mais velhos e, em conseqüência, mais pesados na data da desmama.

Apesar do confundimento entre idade da vaca e época de nascimento, no caso das novilhas, estes resultados estão de acordo com os descritos por PONS (1989) e PONS et al. (1989), para escores de conformação na raça Hereford. O mesmo ocorre em relação ao ganho em peso do nascimento à desmama (CAMPOS et al., 1989; BARCELOS e LOBATO, 1992; FRIES, 1996b; PAZ, 1997). Os escores mais elevados de C, P e M ocorreram para animais nascidos a partir da segunda quinzena do mês de agosto.

A média observada para idade à desmama foi de 196,04 dias com desvio-padrão de 13,49 dias. A idade mínima à desmama foi de 141 dias e a máxima, de 259 dias. Animais desmamados mais velhos apresentaram os melhores escores de C, P e M.

As soluções de quadrados mínimos, de acordo com a idade do bezerro à desmama, podem ser observadas nas Figuras 7, 8 e 9, respectivamente 


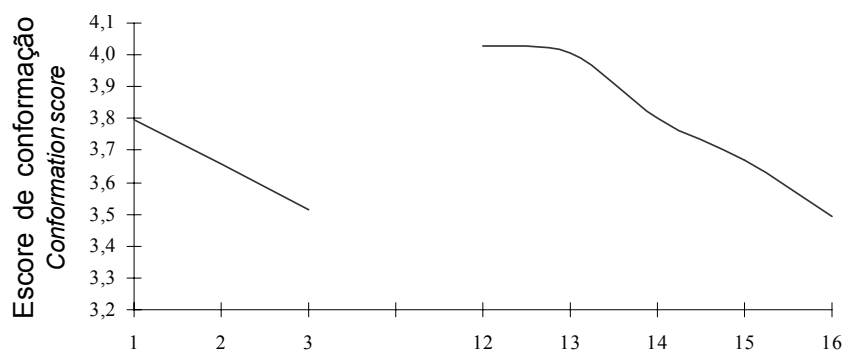

Classe de data juliana de nascimento Julian date of birth class

Figura 4 - Soluções de quadrados mínimos dos escores de conformação de bezerros $(+3,29)$, de acordo com a classe de data juliana de nascimento.

Figure 4 - Least square solutions for conformation scores of calves (+3.29), according to class of Julian date of birth.

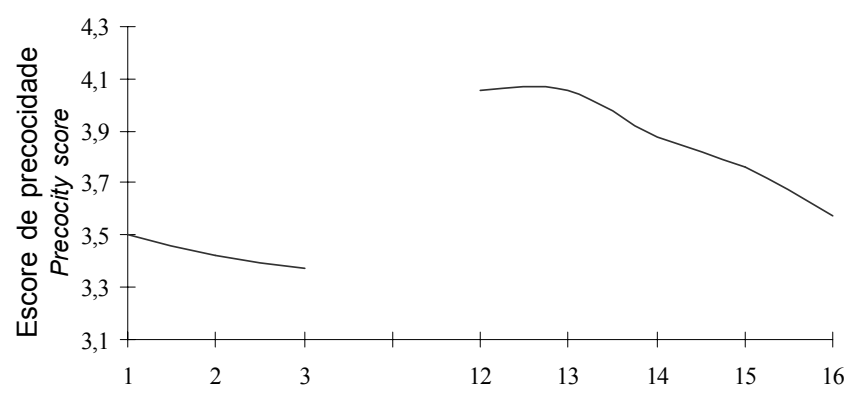

Classe de data juliana de nascimento Julian date of birth class

Figura 5 - Soluções de quadrados mínimos dos escores de precocidade de bezerros $(3,39)$, de acordo com a classe de data juliana de nascimento.

Figure 5 - Least square solutions for precocity scores of calves (+3.39), according to class of Julian date of birth.

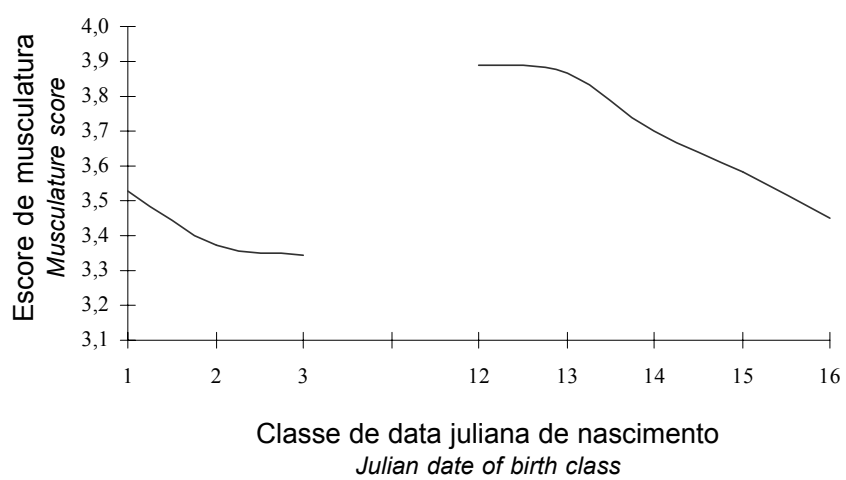

Figura 6 - Soluções de quadrados mínimos dos escores de musculatura de bezerros $(+3,27)$, de acordo com a classe de data juliana de nascimento.

Figure 6 - Least square solutions for musculature scores of calves (+3.27), according to class of Julian date of birth.

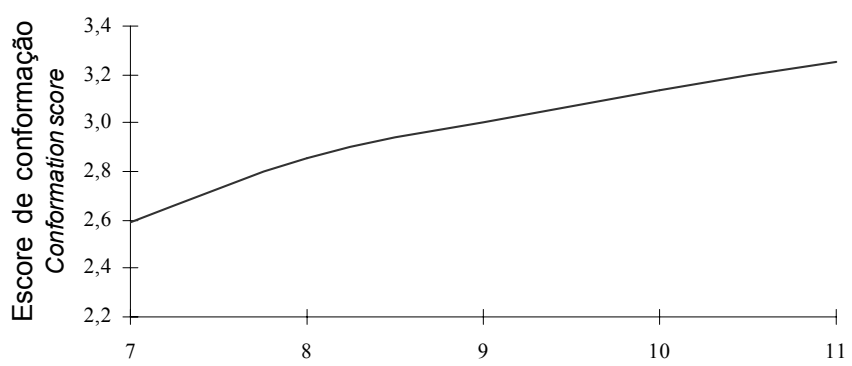

Classe de idade do bezerro à desmama Age of calf at weaning (classes)

Figura 7 - Soluções de quadrados mínimos dos escores de conformação de bezerros $(+3,29)$, de acordo com a classe de idade do bezerro à desmama.

Figure 7 - Least square solutions for conformation scores of calves (+3.29), according to class of age of calf at weaning.

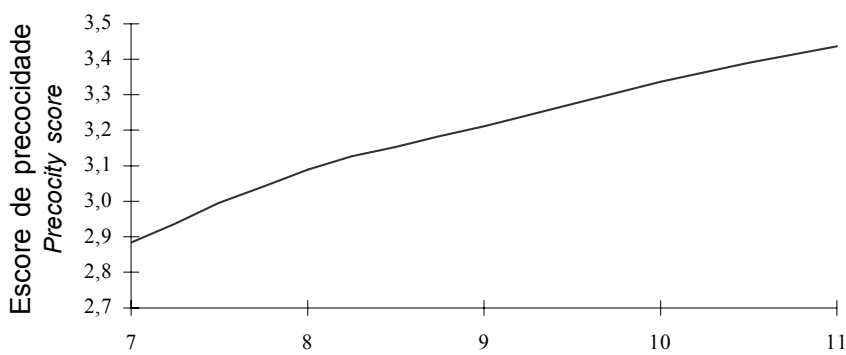

Classe de idade do bezerro à desmama Age of calf at weaning (classes)

Figura 8 - Soluções de quadrados mínimos para os escores de precocidade de bezerros $(+3,39)$, de acordo com a classe de idade do bezerro à desmama.

Figure 8 - Least square solutions for precocity scores of calves (+3.39), according to class of age of calf at weaning.

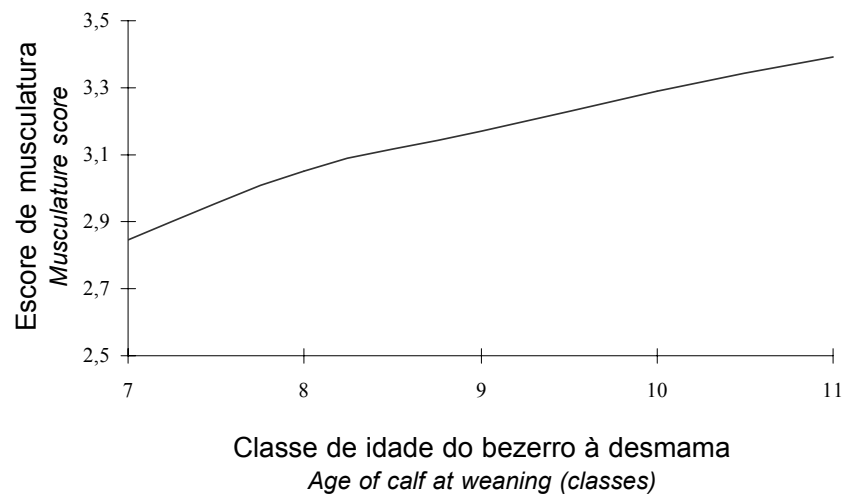

Figura 9 - Soluções de quadrados mínimos para os escores de musculatura de bezerros $(+3,27)$, de acordo com a classe de idade do bezerro à desmama.

Figure 9 - Least square solutions for musculature scores of calves $(+3.27)$, according to class of age of calf at weaning. 
para C, P e M. Os escores mais elevados foram dos animais que desmamaram com 220 dias, enquanto os mais baixos foram dos animais que desmamaram aos 140 dias. Esta é a tendência que ocorre com o peso à desmama, pois, em geral, quanto mais velho o animal à desmama maior o seu peso, ao contrário do que ocorre com o ganho de peso do nascimento à desmama, que diminui com o aumento da idade à desmama (PAZ, 1997).

\section{Conclusões}

Os dados analisados neste trabalho permitiram concluir que a idade da vaca ao parto, a época de nascimento e a idade do bezerro à desmama influenciam significativamente os escores visuais de conformação, precocidade e musculatura, de modo que uma maior precisão na avaliação genética de indivíduos jovens poderá ser alcançada, corrigindo-se previamente os escores originais para esses efeitos.

Estes resultados preliminares revelam a importância de que estudos mais completos e sofisticados, com maiores conjuntos de dados, envolvendo vários rebanhos e com o uso de modelos menos parcimoniosos, sejam utilizados.

\section{Agradecimento}

Ao Sr. Ian David Hill e à Agropecuária Jacarezinho Ltda., pelo fornecimento dos dados.

\section{Referências Bibliográficas}

BARCELOS, J.O.J., LOBATO, J.F.P. 1992. Efeitos da época de nascimento no desenvolvimento de bezerros Hereford e suas cruzas. I. Peso ao nascer e ganho médio diário pré desmama. R. Soc. Bras. Zootec., 21(1):137-149.

CAMPOS, L.T., SILVA P.R., FRIES, L.A. 1989. Fatores de correção para efeitos ambientais que afetam o ganho de peso do nascimento à desmama em bovinos da raça Nelore. Coletânea de pesquisas inéditas sobre zebu. Empresa de Pesquisa Agropecuária de Minas Gerais. Uberaba, MG. p.8-123.

CARDOSO, F.F., CARDELLINO, R.A., CAMPOS, L.T. Efeitos ambientais sobre caracteres produtivos à desmama de bovinos da raça Santa Gertrudis. In: CONGRESSO DE MEDICINA VETERINÁRIA DO CONE SUL, 2., 1997, Gramado. Anais... Gramado, 1997. p.256.
FRIES, L.A. Efeito da idade da vaca sobre o peso à desmama em zebuínos. In: SIMPÓSIO NACIONAL DE MELHORAMENTO ANIMAL, 1, 1996, Ribeirão Preto. Anais... Ribeirão Preto: SBMA, 1996a. p.255-256.

FRIES, L.A. Efeito da data juliana sobre o peso à desmama em zebuínos. In: SIMPÓSIO NACIONAL DE MELHORAMENTO ANIMAL, 1, 1996, Ribeirão Preto. Anais... Ribeirão Preto: SBMA, 1996b. p.249-251.

LONG, R.A. 1973. El sistema de evaluacion de Ankony y su aplicacción en la mejora del ganado. Colorado: Ankony Corporation, Grand Junction.

MOREIRA, H.L.M., CARDELLINO, R.A. 1994. Herdabilidade e repetibilidade e efeitos ambientais em bovinos Hereford. Pesq. Agropec. Bras., 29(1):1795-1800.

PAZ, C.C.P. Efeitos ambientais e genéticos que afetam o ganho de peso de pré desmama em bovinos da raça Nelore. Jaboticabal, SP: UNESP, 1997. 117p. Dissertação (Mestrado em Zootecnia) - Faculdade de Ciências Agrárias e Veterinárias de Jaboticabal, Universidade Estadual Paulista, 1997.

PONS, S.B. Efeitos de fatores genéticos e de ambiente sobre o crescimento e escore de conformação em bovinos da raça Hereford no RS. Viçosa, MG: UFV, 1989. Dissertação (Doutorado em Zootecnia) - Universidade Federal de Viçosa, 1989.

PONS, S.B., MILAGRES, J.C., TEIXEIRA, N.M. 1989. Efeitos de fatores genéticos e de ambiente sobre o crescimento e escore de conformação em bovinos da raça Hereford no RS. R. Soc. Bras. Zootec.,18(5):391-401.

SAS Institute Inc., SAS / STAT. User's Guide, v. 6.11 ed. Cary: SAS INSTITUTE. INC., 1995. 530p.
Recebido em: 08/08/00 Aceito em: 05/06/01 